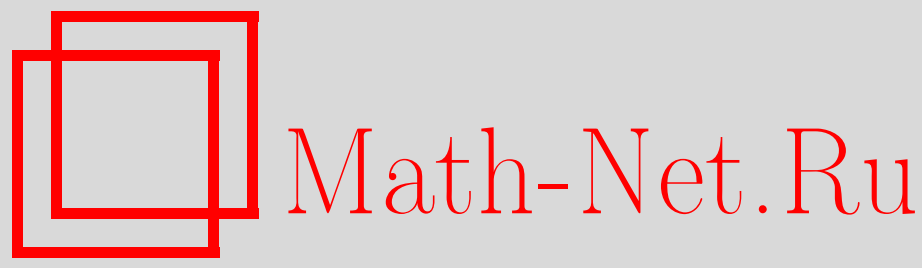

Ф. Ахмед, Осесимметричное пространство-время чистого излучения с геодезическими, нарушающими причинность, ТМФ, 2018, том 195, номер 3, 483-490

DOI: https://doi.org/10.4213/tmf9450

Использование Общероссийского математического портала Math-Net.Ru подразумевает, что вы прочитали и согласны с пользовательским соглашением http://www . mathnet.ru/rus/agreement

Параметры загрузки:

IP : 3.91 .87 .62

26 апреля 2023 г., $12: 23: 49$

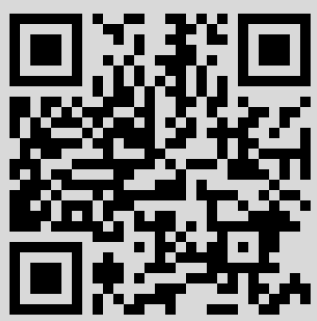




\title{
ОСЕСИММЕТРИЧНОЕ ПРОСТРАНСТВО-ВРЕМЯ ЧИСТОГО ИЗЛУЧЕНИЯ С ГЕОДЕЗИЧЕСКИМИ, НАРУШАЮЩИМИ ПРИЧИННОСТЬ
}

\begin{abstract}
Представлено стационарное осесимметричное пространство-время, допускающее круговые замкнутые времениподобные геодезические всюду в конечной области пространства. Такое пространство-время свободно от расходимостей кривизны и локально изометрично невакуумному рр-волновому пространствувремени. Материя-энергия представляет собой чистое поле излучения и удовлетворяет условию нулевой энергии, а метрика принадлежит к классу $\mathrm{N}$ в классификации Петрова. Наконец,показано, что для данной метрики существуют времениподобные и нулевые геодезические.
\end{abstract}

Ключевые слова: невакуумное решение, нарушение причинности, поля излучения, рр-волны.

DOI: https://doi.org/10.4213/tmf9450

\section{1. ВВЕДЕНИЕ}

Хорошо известны решения полевых уравнений Эйнштейна, в которых имеются кривые, нарушающие причинность, и которые, следовательно, противоречат принципу причинности в общей теории относительности. Первое из таких решений с замкнутыми времениподобными кривыми (ЗВК) и замкнутыми нулевыми кривыми, но без замкнутых времениподобных геодезических (ЗВГ) было найдено в статье [1] это Вселенная Гёделя. После этого и вплоть до сегодняшнего дня было найдено несколько решений, допускающих ЗВК (см. работы [2]-[6] и ссылки в них). Кроме того, некоторые решения допускают ЗВГ и замкнутые нулевые геодезические. Малую часть таких решений можно найти в работах [7]-[10]. В цилиндрически-симметричных пространствах-временах ЗВГ образуются всюду вне осей симметрии [11], а также внутри конечной области пространства [12]. Одним из способов классифицировать подобные нарушающие причинность пространства-времена могло бы быть соотнесение метрики с одним из двух типов: либо с вечной машиной времени, в которой ЗВК или другие кривые формируются повсюду или изначально существуют [1],

${ }^{*}$ Ajmal College of Arts and Science, Dhubri, Assam, India.

E-mail: faizuddinahmed15@gmail.com 
либо с машиой времени, в которой ЗВК возникают по прошествии некоторого периода [3]. Геодезические - это мировые линии свободных частиц. Пространство-время машины времени из работ [13], [14] нарушает слабое энергетическое условие и/или в нем присутствует сингулярность кривизны [15].

\section{2. СТАЦИОНАРНАЯ ОСЕСИММЕТРИЧНАЯ МЕТРИКА}

Наиболее простой интервал в стационарном осесимметричном пространстве-времени $\left(\right.$ с $\left.x^{1}=r, x^{2}=\phi, x^{3}=z, x^{4}=t\right)$ задается как

$$
d s^{2}=g_{r r} d r^{2}+d z^{2}+g_{\phi \phi} d \phi^{2}+2 d t d \phi+2 g_{r \phi} d r d \phi
$$

где метрические функции суть

$$
g_{r r}=e^{2 \alpha r}, \quad g_{\phi \phi}=e^{\alpha r}\left(r^{2}-\alpha r\right), \quad g_{r \phi}=z e^{\alpha r},
$$

a $\alpha>0$ есть действительное число. Координаты меняются в следующих пределах:

$$
0 \leqslant r<\infty, \quad \phi \in[0,2 \pi), \quad z \in(-\infty,+\infty), \quad t \in(-\infty,+\infty) .
$$

Данная метрика имеет сигнатуру $(+,+,+,-)$, а определитель $\operatorname{det} g=-e^{2 \alpha r}$ соответствующего метрического тензора $g_{\mu \nu}$ регулярен даже при $r=0$. Метрика (1) является невакуумным решением со скаляром Риччи $R=0$ и единственной ненулевой компонентой $R_{\mu \nu}$ тензора Риччи:

$$
R_{\phi \phi}=\frac{1}{2} e^{-\alpha r}\left(\alpha^{2}-2-2 \alpha r+e^{\alpha r}\right) .
$$

Пространство-время, которое мы изучаем, свободно от расходимостей кривизны, поскольку инварианты скалярной кривизны, построенные из тензора Римана, равны нулю, $R_{\mu \nu \rho \sigma} R^{\mu \nu \rho \sigma}=0$ и $R_{\mu \nu \rho \sigma} R^{\rho \sigma \lambda \tau} R_{\lambda \tau}^{\mu \nu}=0$.

Полевые уравнения Эйнштейна (при $R=0$ и космологической постоянной, взятой как $\Lambda=0$ ) имеют вид $R_{\mu \nu}=T_{\mu \nu}, \mu, \nu=1,2,3,4$, где выбрана система единиц, в которой $c=1,8 \pi G=1$. Тензор энергии-импульса для полей излучения задается каK [18]

$$
T_{\mu \nu}=\rho k_{\mu} k_{\nu}, \quad k_{\mu} k^{\mu}=0,
$$

где плотность энергии

$$
\rho=\frac{1}{2} e^{-\alpha r}\left(\alpha^{2}-2-2 \alpha r+e^{\alpha r}\right), \quad 0 \leqslant r<\infty,
$$

при нулевом векторе $k^{\mu}=\delta_{t}^{\mu}=(0,0,0,1)$. Таким образом, материю-энергию при $\alpha^{2} \geqslant 2$ можно описать как поля излучения с положительной плотностью энергии.

Метрика (1) является аксиально-симметричной, что ясно из следующих рассуждений. Рассмотрим вектор Киллинга $\eta=\partial_{\phi}$, нормальная форма которого такова:

$$
\eta^{\mu}=(0,1,0,0)
$$

Его ковектор задается как

$$
\eta_{\mu}=\left(z e^{\alpha r}, e^{\alpha r}\left(r^{2}-\alpha r\right), 0,1\right)
$$


Вектор (7) удовлетворяет уравнению Киллинга $\eta_{\mu ; \nu}+\eta_{\nu ; \mu}=0$, где точкой с запятой обозначено ковариантное дифференцирование. Аксиально-симметричное пространство-время (1) задается условием [17]

$$
\mathbf{X} \equiv\left\|\partial_{\phi}\right\|^{2}=\left|g_{\mu \nu} \eta^{\mu} \eta^{\nu}\right|=\left|e^{\alpha r}\left(r^{2}-\alpha r\right)\right| \rightarrow 0, \quad r \rightarrow+0,
$$

где радиальная координата $r$ отсчитывается так, что ось проходит через точку $r=0$. Помимо этой симметрии, метрика обладает симметрией относительно одновременного отражения (инверсии в терминологии Чандрасекара [19]) $(t, \phi) \rightarrow(-t,-\phi)$. В результате (1) известна как круговая стационарная осесимметричная метрика.

\section{3. КРИВЫЕ, НАРУШАЮЩИЕ ПРИЧИННОСТЬ}

В данном разделе сначала мы обсуждаем кривые, нарушающие причинность, а именно ЗВК и ЗВГ в пространстве-времени.

3.1. Рассмотрим азимутальную кривую $\gamma$, задающуюся уравнениями $r=$ const, $z=$ const и $t=$ const. Из выражения для метрики (1) имеем

$$
d s^{2}=g_{\phi \phi} d \phi^{2}=e^{\alpha r}\left(r^{2}-\alpha r\right) d \phi^{2} .
$$

Такие кривые являются нулевыми при $r=r_{0}$, где $r_{0}=\alpha$, и пространственноподобными при $r>r_{0}$, но при $r<r_{0}$ они становятся времениподобными, что показывает присутствие ЗВК. Следовательно, такие кривые образуются в конечной области пространства, при $r<r_{0}$, и в этой области азимутальная компонента метрики $g_{\phi \phi}$ также времениподобна. Любая точка области $0<r<r_{0}$ принадлежит какой-либо ЗВК, но это неверно ни для одной из точек области $r>r_{0}$, поскольку область $r>r_{0}$ расслоена пространственноподобными гиперповерхностями $t=$ const, если $g_{\phi \phi}>z^{2}$. Чтобы показать, что полученные выше ЗВК являются ЗВГ, применим два подхода, обсуждавшихся в работах [9] и [10], [11] соответственно.

МЕтод 1. Применим технику, принятую в работе [9], чтобы показать существование ЗВГ в пространстве-времени.

Квадрат длины вектора $\hat{e}_{\phi}=\partial / \partial \phi$, касательного к определенной выше азимутальной кривой $\gamma$, равен $(\partial / \partial \phi)^{2}=g_{\phi \phi}$, а 4-вектор ускорения $A^{\mu}$ задается как

$$
A^{\mu}=U_{; \nu}^{\mu} U^{\nu}
$$

Для времениподобного единичного вектора (скорости пробных частиц, движущихся по круговым траекториям)

$$
g_{\mu \nu} U^{\mu} U^{\nu}=-1 \quad \Longrightarrow \quad g_{\phi \phi} U^{\phi} U^{\phi}=-1 \quad \Longrightarrow \quad U^{\mu}=\delta_{\phi}^{\mu}\left[-g_{\phi \phi}\right]^{-1 / 2},
$$

где круговые орбиты времениподобны, $g_{\phi \phi}<0$.

Из выражения (11) получаем компоненты ускорения:

$$
\begin{gathered}
A^{r}=\Gamma_{\phi \phi}^{r}\left(U^{\phi}\right)^{2}=\frac{e^{-2 \alpha r} g_{\phi \phi}^{\prime}}{2 g_{\phi \phi}}, \quad A^{\phi}=0, \\
A^{z}=0, \quad A^{t}=\Gamma_{\phi \phi}^{t}\left(U^{\phi}\right)^{2}=-\frac{e^{-\alpha r} z g_{\phi \phi}^{\prime}}{2 g_{\phi \phi}},
\end{gathered}
$$


где штрихом обозначена производная по $r$. Поскольку $U_{; \nu}^{\mu} U^{\nu}=0$ являются геодезическими, решение уравнения $A^{\mu}=0$ относительно $r$ дает геодезическую, т. е. $g_{\phi \phi}^{\prime}\left(r_{*}\right)=0$ при $r=r_{*}<r_{0}$. Ускорение $A$, задающееся формулой $A^{2}=A^{\mu} A_{\mu}$, равно

$$
A=\frac{1}{2} e^{-\alpha r} \frac{g_{\phi \phi}^{\prime}(r)}{g_{\phi \phi}(r)}
$$

оно обращается в нуль при $r=r_{*}$, таким образом, кривая $\gamma$ - это геодезическая.

МЕтод 2. Применим другую технику, представленную в работах [10], [11], чтобы показать существование ЗВГ в пространстве-времени. Продифференцируем по $r$ выражение для азимутальной метрической компоненты

$$
g_{\phi \phi}=e^{\alpha r}\left(r^{2}-\alpha r\right)
$$

получим

$$
g_{\phi \phi}^{\prime}=e^{\alpha r}\left[\alpha r^{2}-r\left(\alpha^{2}-2\right)-\alpha\right] .
$$

Решим уравнение $g_{\phi \phi}^{\prime}=0$ относительно $r$ (выбрав положительный корень):

$$
r=r_{*}=\frac{1}{2 \alpha}\left[\left(\alpha^{2}-2\right)+\sqrt{4+\alpha^{4}}\right] .
$$

Подставив $r=r_{*}<r_{0}$ в формулу (15), нетрудно показать, что условия $g_{\phi \phi}\left(r_{*}\right)<0$ и $\left(g_{\phi \phi}\left(r_{*}\right)\right)_{, z}=0$ гарантируют существование ЗВГ в пространстве-времени (1). Таким образом, указанные выше ЗВК являются ЗВГ при $r=r_{*}<r_{0}$. Поскольку в пространстве-времени (1) существуют ЗВГ, функция $s(r)=2 \pi\left|g_{\phi \phi}(r)\right|^{1 / 2}$ достигает локального максимума при некотором значении $r=\bar{r}, 0<\bar{r} \leqslant r_{*}$, и при этом мы имеем неравенство $g_{\phi \phi}(\bar{r})<0$.

\section{4. АНАЛИТИЧЕСКОЕ ИССЛЕДОВАНИЕ ПРОСТРАНСТВА-ВРЕМЕНИ}

Для метрики (1) получаем следующий лагранжиан:

$$
\mathcal{L}=\frac{1}{2} g_{\mu \nu} \dot{x}^{\mu} \dot{x}^{\nu}=\frac{1}{2}\left[e^{2 \alpha r} \dot{r}^{2}+\dot{z}^{2}+g_{\phi \phi} \dot{\phi}^{2}+2 \dot{t} \dot{\phi}+2 z e^{\alpha r} \dot{r} \dot{\phi}\right],
$$

где точкой обозначено дифференцирование по собственному времени $\tau$. Ясно, что $t$ и $\phi$ являются циклическими координатами, и существуют отвечающие им интегралы движения: один $p_{t}=-E$ и другой $p_{\phi}=L$. Радиальная компонента уравнения геодезической в плоскости $z=$ const $=0$ записывается как

$$
e^{2 \alpha r}\left(\ddot{r}+\alpha \dot{r}^{2}\right)-\frac{1}{2} g_{\phi \phi}^{\prime} \dot{\phi}^{2}=0 .
$$

Для круговых геодезических с $\ddot{r}=0$ и $\dot{r}=0$ мы должны иметь $g_{\phi \phi}^{\prime}=0$. Поскольку $\phi$ есть циклическая координата, имеем $p_{\phi}=g_{\phi \phi} \dot{\phi}+\dot{t}=$ const. Для $\dot{t}=0$, т. е. для замкнутых орбит в пространстве-времени, момент импульса $g_{\phi \phi} \dot{\phi}=$ const, и из (18) в случае круговых ЗВГ мы имеем $g_{\phi \phi} \dot{\phi}^{2}=-1$, откуда следует, что вдоль ЗВГ компонента метрики $g_{\phi \phi}<0$ и $\dot{\phi}=$ const. 
Гамильтониан $H$ для метрики (1) задается как

$$
H=\frac{1}{2} g^{\mu \nu} p_{\mu} p_{\nu}=\frac{1}{2}\left[e^{-2 \alpha r} p_{r}^{2}+p_{z}^{2}-2 z e^{-\alpha r} p_{r} p_{t}+2 p_{t} p_{\phi}+\left(z^{2}-g_{\phi \phi}\right) p_{t}^{2}\right],
$$

при этом $H=-1 / 2$ для времениподобных геодезических и $H=0$ для нулевых (светоподобных).

СлУчАй 1: угловая скорость наблюдателя с нулевым моментом импульса.

Из гамильтониана (20) получаем гамильтоновы уравнения

$$
\dot{t} p_{\phi}+\left[z^{2}-g_{\phi \phi}\right] p_{t}-z e^{-\alpha r} p_{r}, \quad \dot{\phi}=p_{t} .
$$

Отсюда

$$
\Omega\left(r, z, p_{t}, p_{r}, p_{\phi}\right)=\frac{d \phi}{d t}=\frac{\dot{\phi}}{\dot{t}}=\frac{p_{t}}{p_{\phi}+z^{2} p_{t}-g_{\phi \phi} p_{t}-z e^{-\alpha r} p_{r}} .
$$

Если положить $p_{\phi}=0$, то мы получим угловую скорость $\Omega_{0}$ наблюдателя с нулевым моментом импульса (т. е. частицы с нулевым моментом импульса, измеренным наблюдателем, для которого $t$ - собственное время), которая представляет собой угловую скорость увлечения системы отсчета [20], [21] и имеет вид

$$
\Omega_{0}\left(r, z, E, p_{r}\right)=\frac{p_{t}}{z^{2} p_{t}-g_{\phi \phi} p_{t}-z e^{-\alpha r} p_{r}}=\frac{E}{z^{2} E-E e^{\alpha r}\left(r^{2}-r \alpha\right)+z e^{-\alpha r} p_{r}},
$$

при этом $\Omega_{0} \rightarrow 0$, когда или $r$, или $z$ стремится к бесконечности. Мы выбрали плоскость $z=$ const $=0$, при этом угловая скорость наблюдателя с нулевым моментом импульса равна $\Omega_{0}=-e^{-\alpha r} /\left(r^{2}-\alpha r\right)$; она стремится к нулю при $r \rightarrow \infty$ и меняет знак: $\Omega_{0}>0$ при $r<r_{0}=\alpha$ и $\Omega_{0}<0$ при $r>r_{0}=\alpha$.

СлучАй 2: времениподобные (нулевые) геодезические с $\dot{t}<0$ (с $\dot{t}=0$ соответственно) при ненулевом моменте импульса.

Из гамильтониана (20) получаем гамильтоновы уравнения:

$$
\begin{aligned}
& -\dot{p_{r}}=-\alpha e^{-2 \alpha r} p_{r}^{2}+\alpha z e^{-\alpha r} p_{r} p_{t}-\frac{1}{2} g_{\phi \phi}^{\prime} p_{t}^{2}, \\
& -\dot{p_{z}}=z p_{t}^{2}-e^{-\alpha r} p_{r} p_{t} .
\end{aligned}
$$

Если положить $p_{r}=0, p_{z}=0$, то мы получаем

$$
-\dot{p_{r}}=-\frac{1}{2} g_{\phi \phi}^{\prime} p_{t}^{2}, \quad-\dot{p}_{z}=z p_{t}^{2}
$$

Приравнивая правые части этих уравнений к нулю, мы выводим следующие решения: $z=0$ и $g_{\phi \phi}^{\prime}(r)=0$, поскольку $p_{t} \neq 0$, следовательно,

$$
r=r_{*}=\frac{1}{2 \alpha}\left[\left(\alpha^{2}-2\right)+\sqrt{\alpha^{4}+4}\right] .
$$

Таким образом, мы получили обсуждавшееся выше условие существования ЗВГ для метрики (1). В случае времениподобных геодезических введем функцию $K$ (так называемую функцию Картера) формулой

$$
H+\frac{1}{2}=\frac{1}{2}\left[e^{-2 \alpha r} p_{r}^{2}+p_{z}^{2}-2 z e^{-\alpha r} p_{r} p_{t}\right]+\frac{1}{2} K=0,
$$


то есть

$$
K=2 p_{t} p_{\phi}+\left[z^{2}-g_{\phi \phi}\right] p_{t}^{2}+1 .
$$

При $p_{r}=0, p_{z}=0$ и $r=r_{*}<r_{0}$ получаем гамильтоновы уравнения

$$
\dot{\phi}=p_{t}=-E, \quad \dot{t}=p_{\phi}-g_{\phi \phi}\left(r_{*}\right) p_{t}=L-\left|g_{\phi \phi}\left(r_{*}\right)\right| E,
$$

поскольку $g_{\phi \phi}\left(r_{*}\right)<0$, и уравнение $\left|g_{\phi \phi}\left(r_{*}\right)\right| E^{2}-2 E L+1=0$, следовательно,

$$
E_{ \pm}=\frac{1}{\left|g_{\phi \phi}\left(r_{*}\right)\right|}\left[L \pm \sqrt{L^{2}-\left|g_{\phi \phi}\left(r_{*}\right)\right|}\right]
$$

откуда момент импульса $L=p_{\phi} \neq 0$.

В плоскости $z=0$ круговые времениподобные геодезические с $\dot{t}<0$ существуют при (мы выбираем знак плюс для $E) L>\sqrt{\left|g_{\phi \phi}\left(r_{*}\right)\right|}$ и $E>1 / L$.

Для нулевых (светоподобных) геодезических с $\dot{t}=0$, где теперь точкой обозначено дифференцирование по аффинному параметру, мы имеем $L=\sqrt{\left|g_{\phi \phi}\left(r_{*}\right)\right|}$ и $E=1 / L$. Таким образом, метрика (1) допускает существование времениподобных и нулевых круговых геодезических в окрестности оси $z$ на гиперповерхности с $\dot{t} \leqslant 0$ в плоскости $z=$ const.

\section{5. КЛАССИФИКАЦИЯ ПРОСТРАНСТВ}

Построим набор нулевых векторов тетрады $(\mathbf{k}, \mathbf{l}, \mathbf{m}, \overline{\mathbf{m}})$ для метрики (1):

$$
\begin{gathered}
k_{\mu}=(0,1,0,0), \quad l_{\mu}=\left(-z e^{\alpha r},-\frac{e^{\alpha r}}{2}\left(r^{2}-r \alpha\right), 0,-1\right), \\
m_{\mu}=\frac{1}{\sqrt{2}}\left(e^{\alpha r}, 0, i, 0\right), \quad \bar{m}_{\mu}=\frac{1}{\sqrt{2}}\left(e^{\alpha r}, 0,-i, 0\right),
\end{gathered}
$$

где $i=\sqrt{-1}$. В терминах этих векторов метрический тензор интервала (1) можно записать в виде

$$
g_{\mu \nu}=-k_{\mu} l_{\nu}-l_{\mu} k_{\nu}+m_{\mu} \bar{m}_{\nu}+\bar{m}_{\mu} m_{\nu} .
$$

Векторы (31) являются нулевыми и ортогональными, за исключением $k_{\mu} l^{\mu}=-1$ и $m_{\mu} \bar{m}^{\mu}=1$. Ортонормированный базис $\mathbf{e}_{(a)}=\left\{\mathbf{e}_{(1)}, \mathbf{e}_{(2)}, \mathbf{e}_{(3)}, \mathbf{e}_{(4)}\right\}$ выражается через векторы тетрады следующим образом:

$$
\mathbf{k}=\frac{1}{\sqrt{2}}\left(\mathbf{e}_{(4)}+\mathbf{e}_{(2)}\right), \quad \mathbf{l}=\frac{1}{\sqrt{2}}\left(\mathbf{e}_{(4)}-\mathbf{e}_{(2)}\right), \quad \mathbf{m}=\frac{1}{\sqrt{2}}\left(\mathbf{e}_{(1)}+i \mathbf{e}_{(3)}\right) .
$$

Используя векторы (31), находим пять скаляров Вейля, из которых только один отличен от нуля:

$$
\Psi_{0}=\Psi_{1}=\Psi_{2}=\Psi_{3}=0, \quad \Psi_{4}=\frac{1}{4} e^{-\alpha r}\left(\alpha^{2}-2-2 r \alpha\right) .
$$

Метрика (1) имеет тип N в классификации Петрова.

Мы видим, что наша метрика допускает ковариантно постоянный нулевой вектор $k_{\mu}$, т. е. $k_{\mu ; \nu}=0$. Такое пространство-время называется пространством гравитационных волн с плоским фронтом и параллельными лучами (рр-волновое пространство-время). Используя тетраду (31), найдем оптические скалярь [18]: расширение 
(экспансию), вращение (твист) и сдвиг:

$$
\boldsymbol{\Theta}=\frac{1}{2} k_{; \mu}^{\mu}=0, \quad \boldsymbol{\omega}^{2}=\frac{1}{2} k_{[\mu ; \nu]} k^{\mu ; \nu}=0, \quad \boldsymbol{\sigma} \overline{\boldsymbol{\sigma}}=\frac{1}{2} k_{(\mu ; \nu)} k^{\mu ; \nu}-\boldsymbol{\Theta}^{2}=0 .
$$

Можно показать, что пространство-время, задаваемое метрикой (1), приводится к форме Бринкмана [22] для этой категории пространств.

Рассмотрим преобразование $r \rightarrow \ln (\alpha \chi) / \alpha, t \rightarrow v-z \chi / 2$ и далее преобразование

$$
\chi \rightarrow x \cos \frac{\phi}{2}+y \sin \frac{\phi}{2}, \quad z \rightarrow x \sin \frac{\phi}{2}-y \cos \frac{\phi}{2} .
$$

В результате из метрики (1) получим метрику

$$
d s^{2}=d x^{2}+d y^{2}+2 d v d \phi+G(x, y, \phi) d \phi^{2},
$$

где

$$
\begin{aligned}
G(x, y, \phi)=\frac{1}{\alpha} & \left(x \cos \frac{\phi}{2}+y \sin \frac{\phi}{2}\right) \ln \left(\alpha x \cos \frac{\phi}{2}+\alpha y \sin \frac{\phi}{2}\right) \times \\
& \times\left\{\left(\alpha x \cos \frac{\phi}{2}+\alpha y \sin \frac{\phi}{2}\right)-\alpha^{2}\right\}-\frac{1}{4}\left(x^{2}+y^{2}\right) .
\end{aligned}
$$

Эта метрика приводится к форме Бринкмана [22] для рр-волнового пространствавремени.

\section{6. ЗАКЛЮЧЕНИЕ}

В представленной статье мы обсуждали стационарное аксиально-симметричное пространство-время, допускающее круговые ЗВГ. Метрика пространства локально изометрична метрике невакуумного рр-волнового пространства-времени и свободна от расходимостей кривизны. Мы показали, что ЗВГ в таком пространстве существуют в конечной области, следовательно, можно было бы совершить путешествие во времени в состоянии свободного падения. Многие из известных пространств с ЗВК или ЗВГ имеют такие недостатки, как нарушение энергетических условий и/или сингулярности кривизны, являясь тем самым нефизическими. Наша модель свободна от всех этих проблем, и метрика относится к типу N в классификации Петрова. Также мы показали, что данная метрика допускает круговые времениподобные или нулевые геодезические $\mathrm{c} \dot{t} \leqslant 0$ в плоскости $z=$ const при ненулевом моменте импульса, и, следовательно, вдоль этих геодезических время бежит в обратном направлении.

\section{Список литературы}

[1] K. Gödel, "An example of a new type of cosmological solutions of Einstein's field equations of gravitation", Rev. Modern Phys., 21:3 (1949), 447-450.

[2] F. Ahmed, B. B. Hazarika, D. Sarma, "The anti-de Sitter spacetime as a time machine", Eur. Phys. J. Plus, 131:7 (2016), 230, 3 pp.

[3] F. Ahmed, "Type N Einstein space time machine spacetime", Ann. Phys., 382 (2017), $127-135$. 
[4] F. Ahmed, "A stationary cylindrically symmetric spacetime which admits CTCs and its physical interpretation", Ann. Phys., 386 (2017), 25-33.

[5] F. Ahmed, "Closed timelike curves in type II non-vacuum spacetime", Commun. Theor. Phys., 67:2 (2017), 189-191.

[6] F. Ahmed, "A type D non-vacuum spacetime with causality violating curves, and its physical interpretation", Commun. Theor. Phys., 68:6 (2017), 735-740.

[7] I. D. Soares, "Inhomogeneous rotating universes with closed timelike geodesics of matter", J. Math. Phys., 21:3 (1980), 521-525.

[8] B. R. Steadman, "Letter: Causality violation on van Stockum geodesics", Gen. Rel. Grav., 35:9 (2003), 1721-1726.

[9] W. B. Bonnor, B. R. Steadman, "Exact solutions of the Einstein-Maxwell equations with closed timelike curves", Gen. Rel. Grav., 37:11 (2005), 1833-1844.

[10] Ø. Grøn, S. Johannesen, "Closed timelike geodesics in a gas of cosmic strings", New J. Phys., 10 (2008), 103025, 17 pp.

[11] O. Grøn, S. Johannesen, "A spacetime with closed timelike geodesics everywhere", Nuovo Cimento B, 125:10 (2010), 1215-1221.

[12] F. Ahmed, "Cylindrically symmetric pure radiation space-time and closed timelike geodesics", Progr. Theor. Exp. Phys., 2017:4 (2017), 043E02, 12 pp.

[13] M. S. Morris, K. S. Thorne, U. Yurtsever, "Wormholes, time machines, and the weak energy condition", Phys. Rev. Lett., 61:13 (1988), 1446-1449.

[14] M.S. Morris, K.S. Thorne, "Wormholes in spacetime and their use for interstellar travel: A tool for teaching general relativity", Amer. J. Phys., 56:3 (1988), 395-412.

[15] F. Ahmed, "Gravitational collapse of type N spacetime, the naked singularity, and causality violation", Progr. Theor. Exp. Phys., 2017:8 (2017), 083E03, 12 pp.; "Cylindrically symmetric, asymptotically flat, Petrov type D spacetimewith a naked curvature singularity and matter collapse", Adv. High Energy Phys., 2017 (2017), 7943649; "Axially symmetric null dust spacetime, naked singularity, and cosmictime machine", 3587018.

[16] J. Carot, J. M. M. Senovilla, R. Vera, "On the definition of cylindrical symmetry", Class. Quantum Grav., 16:9 (1999), 3025-3034.

[17] A. Barnes, "A comment on a paper by Carot et al.", Class. Quantum Grav., 17:13 (2000), 2605-2609.

[18] H. Stephani, D. Kramer, M. MacCallum, C. Hoenselaers, E. Herlt, Exact Solutions to Einstein's Field Equation, Cambridge Univ. Press, Cambridge, 2003.

[19] С. Чандрасекар, Математическая теория черных дыр, Мир, М., 1986.

[20] P. Collas, D. Klein, "Letter: Frame dragging anomalies for rotating bodies", Gen. Rel. Grav., 36:5 (2004), 1197-1206.

[21] T.-M. He, Y.-J. Wang, "Frame dragging in the field of Kerr family", Chinese Phys., 15:1 (2006), 232-234.

[22] H. W. Brinkmann, "Einstein spaces which are mapped conformally on each other", Math. Ann., 94:1 (1925), 119-145.

Поступила в редакцию 19.08.2017, после доработки 12.12.2017 